\title{
PERCEPCIONES DE LAS PERSONAS MENORES DE EDAD, QUE FUERON ATENDIDAS EN EL INSTITUTO SOBRE ALCOHOLISMO Y FARMACODEPENDENCIA, RESPECTO A LA MARIHUANA, 2017.
}

\author{
Sáenz Márquez, Kate ${ }^{1}$ \\ ${ }^{1}$ Instituto sobre Alcoholismo y Farmacodependencia, San José, Costa Rica.
}

Resumen: El presente documento indaga sobre las percepciones que los jóvenes tienen sobre la marihuana, pretende un acercamiento real a una consulta, una conversación sobre sus pensamientos. Todos los integrantes tienen más de un año de consumir marihuana en forma activa, y fueron referidos a un programa donde son atendidos, ya que principalmente sus familias han consultado debido a que han visto una problemática biopsicosocial en los jóvenes. A pesar de los riesgos que ellos refieren, éstos no son de peso suficiente para no realizar el consumo. ¿Cómo hablar más allá de que la marihuana "quema neuronas" o que "es planta, pero no es medicinal"? ¿Cómo llegar a empatizar con los jóvenes para dar información relevante para ellos y sus familias en cuanto al consumo de drogas? ¿Cómo mejorar los programas de prevención del consumo de drogas que se les imparten a los niños desde edades tempranas para que no tengan un acercamiento con las drogas y menos un consumo activo de las mismas? Son algunas de las interrogantes que los que trabajan en la promoción de la salud mental y el tratamiento por el consumo de sustancias psicoactivas se hacen.

Palabras clave: percepciones, marihuana, menores, droga, salud mental. Fuente: NLM, Mesh.

Recibido: 30 Junio 2017. Aceptado: 17 Agosto 2017. Publicado: 25 Octubre 2017.

Revista electrónica publicada por el Departamento de Farmacología de la Escuela de Medicina de la Universidad de Costa Rica, 2060 San José, Costa Rica. ${ }^{\circledR}$ All rights reserved. Licensed under a Creative Commons Unported License. 


\title{
PERCEPTIONS OF UNDERAGE PERSONS REGARDING TO MARIJUANA, TREATED IN THE INSTITUTE ON ALCOHOLISM AND DRUG DEPENDENCE, 2017.
}

\begin{abstract}
This document presents perceptions young people have about marijuana, a real approach to a query with minors, a conversation about their thoughts, to know them. All members are patients that have more than one year of active marijuana use. They are referred to a program because families requested treatment as it seems to be a biopsychosocial problem. It is clear that despite the risks they may see, they do not have enough sway for them not to stop using the drug. How to speak to them not just that marijuana "burns neurons"? or that it is a plant but not medicinal? How to empathize with young people to provide them and their families relevant information regarding drug usage? How to improve the early age programs of prevention of drug use that are developed to children so that they do not approach drugs and won't start using them? These are some of the questions that we as professionals investigate while working on the promotion of mental health and treatment of patients who uses psychoactive substances.
\end{abstract}

Key words: perceptions, marijuana, minors, drug, mental healt. Source: NLM, Mesh.

\section{INTRODUCCION}

El objetivo de este documento es reflejar las percepciones con respecto a la marihuana que tienen las personas menores de edad (PME) que consultan al Programa ambulatorio intensivo (PAI) del Centro de Atención Integral en Drogas para Personas Menores del Instituto sobre Alcoholismo y Farmacodependencia (IAFA), Centro de Menores (CM).

Entiéndase percepción, según la Real Academia Española, como: conocimiento, idea, y esto como: noción, saber. Por lo tanto, para este investigación se tomará, como percepción a las ideas o juicios con respecto a la marihuana, esto es importante para determinar aspectos relacionados al consumo, en una primera o segunda entrevista, lo generalmente notado es que una persona no exprese en primera instancia la razón real por la cuál consume marihuana y una PME "puede que no tenga definida esa respuesta", realizar preguntas abiertas al inicio de una entrevista ayuda al terapeuta a entablar un diálogo dirigido, pero no recriminatorio al respecto de la sustancia consumida en cuestión.
En los años de trabajo como médico y como terapeuta de personas consumidoras de drogas surgen algunas interrogantes que se consideran importantes estudiar, una de ellas es conocer lo que piensan las personas menores de edad consumidores de marihuana con respecto a la droga. En los últimos años la gran mayoría de los pacientes tratados por consumo, principalmente de la droga marihuana vienen a las primeras citas con diversos discursos, dentro de los cuales están: "La marihuana es planta", "la marihuana es droga, pero no es mala", "no sé lo que pienso", "la marihuana quema neuronas", entre otras.

La importancia de documentar esta información sobre esta droga es por sus consecuencias mentales, físicas y por ser la segunda droga de más consumo entre los jóvenes, después del tabaco según la Encuesta de colegiales en Costa Rica, IAFA, 2015 ,

Además, dado que la marihuana es una droga que al ser utilizada, puede llevar a un consumo perjudicial, entiéndase como un patrón de consumo que causa daño a la salud y, a que es la 
droga ilícita más consumida por la población adolescente, los resultados de este trabajo pueden ser relevantes para centros y servicios de salud, fortaleciendo proyectos y programas de información, de promoción de la salud, de prevención del consumo de drogas y del tratamiento de los problemas asociados al mismo. La percepción de riesgo puede ser entendida como un juicio un tanto subjetivo, ya que la severidad del dependiendo de los juicios de cada una, por lo cual no es ese el propósito de este artículo. Si es de bajo riesgo o de alto riesgo consumir marihuana a final de cuentas no nos dice mucho sobre el pensamiento de las PME con respecto a la droga, sin embargo las preguntas abiertas sobre la reflexión que pueden hacer alrededor de lo que significa la droga para ellos, además que está presentado en un ambiente de atención integral, donde se ha propiciado la empatía, la comunicación asertiva y sobre todo con un gran respeto sin fines de juicio represor.

Los estudios e investigaciones realizadas sobre la epidemiología del consumo de drogas coinciden en apuntar las relaciones existentes entre las percepciones que los ciudadanos tienen de las distintas sustancias y sus hábitos de consumo, constatándose la existencia de una relación inversa entre el riesgo percibido y la prevalencia de uso de drogas.

El consumo de marihuana representa una prevalencia significativa en nuestro país. Entre la población de educación secundaria, las estadísticas recopiladas por el IAFA muestran un incremento significativo en cuanto al consumo de marihuana en los últimos 12 meses. Para el 2006, el 4,5\% de los estudiantes de secundaria consumieron esta sustancia en este periodo, para el 2009 esa proporción aumenta a 6,8\%, durante el 2012 se registró un 9,7\% y finalmente, para el 2015 se reporta un 9,4\%. En todas las rondas de encuestas, la población masculina presenta un mayor consumo que el femenino, no obstante el consumo en ambos sexos se presenta en aumento constante desde el 2006. riesgo puede cambiar de persona a persona

De igual forma, el consumo activo, o del último mes, entre los estudiantes de secundaria ha reportado un aumento desde el 2006 cuando se estableció en 1,9\%, a un 4,9\% en el 2015. Coincidentemente, la VI Encuesta Nacional sobre consumo de drogas en población general, realizada también durante el 2015, reporta una prevalencia de consumo activo de 4,9\% entre la población de 12 a 19 años, ratificando de esta forma el incremento del consumo en las poblaciones más jóvenes.

Tanto en hombres como en mujeres, la edad promedio de la primera ocasión de consumo de marihuana entre los colegiales alcanzó un promedio de 14,2 años en el 2015, en tanto que en población general ese promedio se ubica en 18,1 años, resaltando así, que el primer contacto se establece durante la adolescencia.

En el Centro Nacional de Atención Integral para Personas Menores de Edad en el 2015, se valoraron un total de 2311 personas menores de edad de las cuales, 1822 adolescentes corresponden al sexo masculino y 489 adolescentes al sexo femenino. En la modalidad de atención ambulatoria intensiva se brindó tratamiento a 364 adolescentes (284 hombres y 80 mujeres), que recibieron 2799 atenciones.

Este artículo, señala las respuestas dadas por $32 \mathrm{de}$ los 145 pacientes activos en el PAI, elegidos aleatoriamente, según datos entregados al equipo PAI a partir de la revisión de expedientes en mayo del 2016, ésta es una muestra pequeña por lo que no permite hacer generalizaciones, vale acotar. Todos los pacientes encuestados pertenecen al programa ambulatorio intensivo y cumplen con los criterios de dependencia a la marihuana. La dependencia se define según la Organización Mundial de la Salud (OMS) como un patrón desadaptativo de consumo de la sustancia que conlleva un deterioro o malestar clínicamente significativos, incluyendo tolerancia y abstinencia.

Para lo anterior, se desarrollaron una serie de preguntas a modo de entrevista de interés de la Revista electrónica publicada por el Departamento de Farmacología de la Escuela de Medicina de la Universidad de Costa Rica, 2060 San José, Costa Rica. ${ }^{\circledR}$ All rights reserved. Licensed under a Creative Commons Unported License.

Contáctenos: rev.med.ucr@gmail.com. Tel: (506) 25-11 4492, Fax: 25-11-4489. 
autora, dada su experiencia en el tema, cuyo fin es identificar ciertas características del consumo de marihuana en la población establecida.

Con respecto a los datos demográficos, de los 32 pacientes entrevistados, 4 eran mujeres, 28 hombres, 2 no estudiaban y 30 si estaban insertos en el sistema educativo. Con un rango de edad desde los 13 a los 17 años.

Los motivos que los jóvenes adujeron para no consumir drogas fueron muy similares en todos los casos. Existen actitudes de aprobación o rechazo hacia el consumo de drogas.

\section{Tabla $n .^{\circ} 1$. Respuestas de los entrevistados}

\begin{tabular}{|c|c|c|c|c|}
\hline Pregunta & Sí (\%) & No $(\%)$ & $\begin{array}{c}\text { NS/NR } \\
(\%)\end{array}$ & $\begin{array}{c}\text { Total } \\
(\%)\end{array}$ \\
\hline 1 ¿Considera usted que la marihuana es una droga? & $\begin{array}{c}26 \\
(81,3 \%)\end{array}$ & $6(18,8 \%)$ & & $32(100 \%)$ \\
\hline 2 ¿Considera usted que la marihuana hace daño a la salud? & $\begin{array}{c}28 \\
(87,5 \%)\end{array}$ & $4(12,5 \%)$ & & $32(100 \%)$ \\
\hline $\begin{array}{l}3 \text { ¿Considera usted que la marihuana hace daño a los } \\
\text { pulmones? }\end{array}$ & $\begin{array}{c}24 \\
(75,0 \%)\end{array}$ & $7(21,9 \%)$ & $1(3,1 \%)$ & $32(100 \%)$ \\
\hline 4 ¿Considera usted que la marihuana hace daño al cerebro? & $\begin{array}{c}30 \\
(93,8 \%)\end{array}$ & $1(3,1 \%)$ & $1(3,1 \%)$ & $32(100 \%)$ \\
\hline 5 ¿Considera usted que la marihuana es una sustancia legal? & $2(6,3 \%)$ & $\begin{array}{c}29 \\
(90,6 \%)\end{array}$ & $1(3,1 \%)$ & $32(100 \%)$ \\
\hline 6 ¿Su familia está preocupada por su consumo? & $\begin{array}{c}31 \\
(96,9 \%)\end{array}$ & & $1(3,1 \%)$ & $32(100 \%)$ \\
\hline
\end{tabular}

Fuente: elaboración propia, 2017.

Con respecto a las 6 preguntas del cuestionario presentamos los datos en la tabla 1, de la cual podemos diferir lo siguiente:

A los interrogados con la pregunta 1, del $81 \%$ que dijo que sí es droga, el 23\% lo considera así, ya que es adictiva, el $15 \%$ debido a que es ilegal, el $11 \%$ debido a que es dañina y el restante menor debido a que es medicinal y no adictiva. $19 \%$ dijo que no es droga, de éstos $33 \%$ cree que no es droga debido a que es medicinal así como el otro 33\% contestó que no es adictiva y el $17 \%$ no sabe por qué no es droga.

A los entrevistados con la pregunta 2, del 87\% que dijo que si hace daño a la salud, el $39 \%$ afirma esto ya que hace daño al cuerpo, el $25 \%$ cree que afecta a las neuronas, muchos de ellos contestando sobre las neuronas con sus propias palabras "las quema". El 32\% no sabe por qué daña la salud, sin embargo, contestó en forma afirmativa, siendo esto al parecer un tanto contradictorio. Un $12 \%$ cree que no daña la salud, sin embargo $50 \%$ de ellos dice que daña el cuerpo, un $25 \%$ no sabe por qué y se hace la anotación que una PME al decir que no daña a la salud comentó que "la marihuana es un amigo".

A los sondeados con la pregunta 3 , del $75 \%$ que contestó que sí, el 33\% cree que hace daño por los químicos que contiene, además que el humo que se inhala daña y contamina los pulmones, $21 \%$ afirma que los daña, ya que da síntomas respiratorios como tos y dificultad para respirar, sobre todo al realizar alguna actividad física. El 22\% dice que no afecta a los pulmones, sin embargo, no sabe o no responde porqué considera esto.

Revista electrónica publicada por el Departamento de Farmacología de la Escuela de Medicina de la Universidad de Costa Rica, 2060 San José, Costa Rica. ${ }^{\circledR}$ All rights reserved. Licensed under a Creative Commons Unported License. 
A los consultados con la pregunta 4, del 94\% que contestó que sí daña al cerebro, el 50\% dice que se pierden neuronas, el 33\% lo afirma pero no sabe por qué, el $10 \%$ dice que afecta la memoria, lo que piensa y sólo piensa en consumir y vale acotar que un paciente dijo que daña el cerebro a largo plazo. El 6\% dijo que no lo daña o que no sabe.

A los entrevistados con la pregunta 5, el 91\% dijo que la marihuana es ilegal, sólo 2 pacientes contestaron lo siguiente, es legal en medicina, pero ilegal en el consumo y el otro dijo que es ilegal en Costa Rica pero es legal en Estados Unidos y en Jamaica.

A los sondeados con la pregunta 6, el 97\% contestó que su familia sí está preocupada por el consumo, de éstos, el 29\% dice que su familia está preocupada, ya que el consumo trae problemas a su familia y al entorno social, el $26 \%$ porque es dañina, un $13 \%$ porque la familia tiene miedo a la adicción, otro $13 \%$ porque les da miedo que termine en las calles y el resto sólo cree que están preocupados, pero no saben el porqué.

La pregunta 7 es una pregunta abierta que permite que las PME expresen su opinión libremente, sin ser preguntas orientadas, sino que refleja el contexto en el que se encuentran con respecto a la droga. En la figura se muestran las respuestas agrupadas en 6 categorías según las opiniones más destacadas. Entiéndase normalizado como que una cosa sea normal o regular y placentera como algo que es muy agradable.

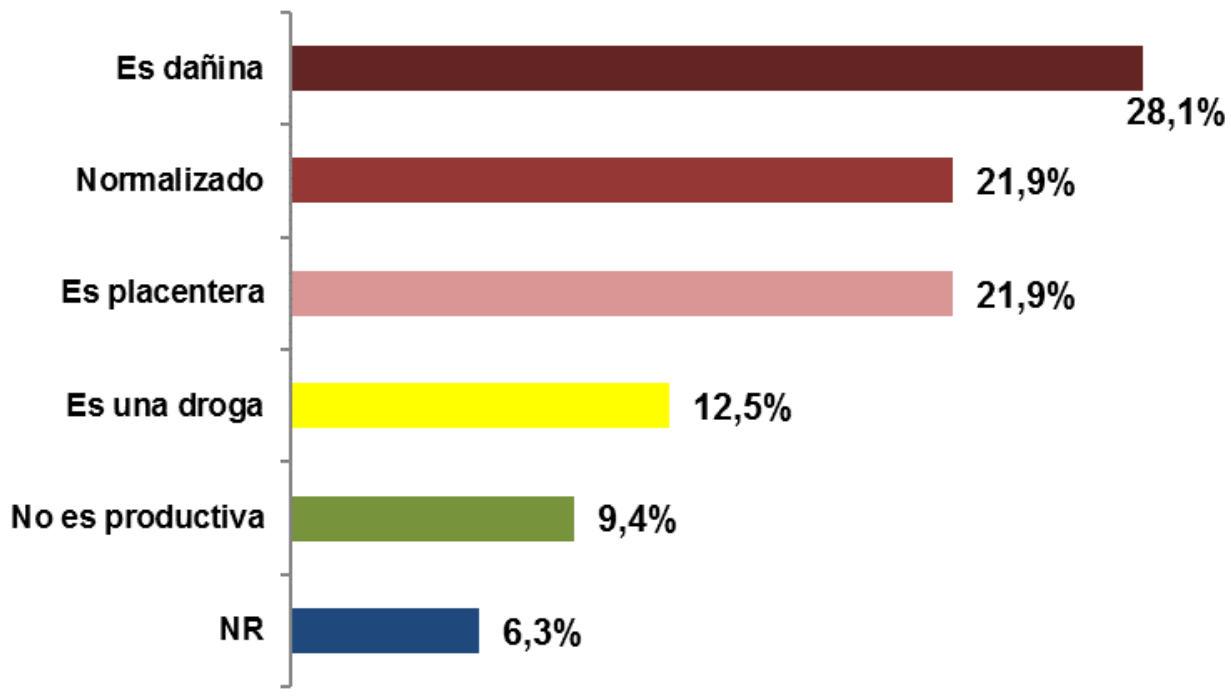

Figura n. ${ }^{\circ}$ 1. Opinión de los entrevistados acerca del consumo de marihuana. Costa Rica, 2016. Fuente: elaboración propia, 2017.

De las PME encuestadas el $44 \%$ refiere que tiene efectos placenteros para ellos y ven la droga como algo normal, dentro de las respuestas que se pueden rescatar están, "me hace sentir bien", "es parte de mí", "me ayuda a dormir", "es medicinal”. Además, el 28\% considera que la marihuana es dañina, dentro de los argumentos están "daña la salud", "afecta los pulmones", "quita los sueños y metas", "daña psicológicamente y físicamente" "lo puede matar a uno". El 12,5\% piensa que es una droga debido a que, "es estimulante y relajante", "lo hace sentir de una manera diferente" "por los químicos que trae hace daño", "genera una adicción". El 9,4\% expresa que no es productiva, 
ya que "es una pérdida de tiempo" y "no deja nada". El $6,3 \%$ no saben qué pensar acerca de la marihuana.

\section{CONCLUSIONES}

Este documento se hace como aporte para orientar acciones que respondan a una política preventiva eficaz en el campo del consumo de drogas en nuestro entorno.

Hay que tener en cuenta que los efectos adversos del consumo de drogas aún no se han producido de forma significativa a esa edad, aunque algunos jóvenes ya han padecido diversos tipos de problemas asociados al uso de drogas.

Sería interesante analizar las percepciones que tienen las PME sobre otras drogas y hacer una comparación al respecto.

Esto nos dirige al grado, la cantidad y calidad de la información que las PME están recibiendo, pese a que la mayoría tiene el concepto que la marihuana afecta en forma biopsicosocial siguen con el consumo.

No siempre una baja percepción de riesgo se asocia a muy altas tasas de consumo de una determinada droga. Un sin número de otros factores inciden para que el uso de una sustancia psicoactiva presente altas o bajas tasas de consumo, por ejemplo, la disponibilidad de la sustancia en el mercado, el precio de la droga, el nivel de aceptación/rechazo social, su capacidad de generar adicción, entre muchos otros.

Aunque, este análisis no permite hacer grandes recomendaciones con respecto a las mejores prácticas en la prevención del uso de drogas, está claro que las percepciones de los jóvenes están asociadas al uso de drogas en diversas poblaciones. Este artículo es un primer avance en el camino de muchas interrogantes que se presentan en la atención a personas consumidoras de drogas y en hacer consciencia sobre las intervenciones, sobre todo de información y preparación en diversas habilidades sociales, que sirvan como

Revista electrónica publicada por el Departamento de Farmacología de la Escuela de Medicina de la Universidad de Costa Rica, 2060 San José, Costa Rica. ${ }^{\circledR}$ All rights reserved. Licensed under a Creative Commons Unported License.

Contáctenos: rev.med.ucr@gmail.com. Tel: (506) 25-11 4492, Fax: 25-11-4489. herramientas para un mundo donde la droga sigue siendo un gran problema de salud pública.

\section{REFERENCIAS}

1. Instituto sobre Alcoholismo y Farmacodependencia. Informe Anual de Cumplimiento 2015. Plan Operativo Institucional Resultado de las Metas 2015. San José, Costa Rica, 2015.

2. Instituto sobre Alcoholismo y Farmacodependencia. Centro de Atención Integral en Drogas para Personas Menores de Edad. Informe Anual de Labores. 2015.

3. Instituto sobre Alcoholismo y Farmacodependencia. Análisis de oferta demanda en la atención de personas menores de edad consumidoras problemáticas de sustancias psicoactivas en Costa

4. Tarter, R. Evaluation and treatment of adolescent substance abuse: A decision tree method. American Journal of Drug and Alcohol Abuse. 1990.

5. Instituto sobre Alcoholismo y Farmacodependencia IAFA. Consumo de Drogas en Costa Rica Encuesta Nacional 2010. Consumo de Cannabis en Costa Rica. Costa Rica, 2012.

6. Instituto sobre Alcoholismo y Farmacodependencia. VI Encuesta Nacional sobre consumo de drogas en

7. Asociación Estadounidense de Psiquiatría. Manual diagnóstico y estadístico de los trastornos mentales. Washington, DC, Estados Unidos, 2000.

8. Instituto sobre Alcoholismo y Farmacodependencia .Política del sector salud para la atención de los problemas derivados del consumo de alcohol, tabaco y otras drogas en Costa Rica. San José, Costa Rica, 2008.

\section{CORRESPONDENCIA}

Sáenz Márquez, Kate

Correo: drkatesaenz@gmail.com Rica 2012, 2014. población general. Costa Rica, 2015. 